\title{
Minimally Invasive Gastric Electrical Stimulation Using a Newly Developed Wireless Gastrostimulator: A Pilot Animal Study
}

\author{
Seung Han Kim, ${ }^{1}$ Hong Bae Kim, ${ }^{2}$ Hoon Jai Chun, ${ }^{\text {* }}$ Hyuk Soon Choi, ${ }^{1}$ Eun Sun Kim, ${ }^{1}$ Bora Keum, ${ }^{1}$ Yeon Seok Seo, ${ }^{1}$ Yoon Tae Jeen, ${ }^{1}$ \\ Hong Sik Lee, ${ }^{1}$ Soon Ho Um, ${ }^{1}$ and Chang Duck Kim ${ }^{1}$ \\ ${ }^{1}$ Division of Gastroenterology and Hepatology, Department of Internal Medicine, Institute of Gastrointestinal Medical Instrument Research, \\ Korea University College of Medicine, Seoul, Korea; and ${ }^{2}$ Department of Biosystems and Biomaterials Science and Engineering, Seoul National \\ University, Seoul, Korea
}

\section{Background/Aims}

Gastric electrical stimulation (GES) is a feasible modality for the treatment of gastroparesis; however, the presently available device requires invasive surgical implantation for long-term stimulation and repeated surgical procedure after a period of time. This study is aimed at developing a wireless miniature GES device and testing its endoscopic insertion in animal models.

\section{Methods}

Endoscopic gastric implantation of the GES device was performed on 5 healthy weaner pigs under general anesthesia. We created an endoscopic submucosal pocket and inserted the gastro-electrical stimulator. In vivo gastric slow waves were recorded and measured during electrical stimulation. A multi-channel recorder, called an electrogastrogram, was used to record the gastric myoelectrical activity in the study.

\section{Results}

The gastric slow waves on the electrogastrogram were more consistent with GES on the gastric tissues compared to no stimulation. The frequency-to-amplitude ratio was also significantly altered after the electrical stimulation.

\section{Conclusions}

GES is feasible with our minimally invasive wireless device. This technique has the potential to increase utilization of GES as a treatment alternative.

(J Neurogastroenterol Motil 2020;26:410-416)

\section{Key Words}

Electric stimulation; Endoscopy; Gastroparesis

Received: April 3, 2020 Revised: April 25, 2020 Accepted: May 10, 2020

(.) This is an Open Access article distributed under the terms of the Creative Commons Attribution Non-Commercial License (http://creativecommons. org/licenses/by-nc/4.0) which permits unrestricted non-commercial use, distribution, and reproduction in any medium, provided the original work is properly cited.

*Correspondence: Hoon Jai Chun, MD, PhD

Division of Gastroenterology and Hepatology, Department of Internal Medicine, Institute of Gastrointestinal Medical Instrument Research, Korea University College of Medicine, 73, Goryeodae-ro, Seongbuk-gu, Seoul 02841, Korea

Tel: +82-2-920-6555, Fax: +82-2-953-1943, E-mail: drchunhj@gmail.com

Seung Han Kim and Hong Bae Kim contributed equally to this work. 


\section{Introduction}

Gastroparesis is a chronic morbid neuromuscular disorder that causes nausea, vomiting, upper abdominal pain, and early satiety. Gastrointestinal symptoms are frequent in gastroparesis and can lead to deterioration of the quality of life. The etiologies of gastroparesis can be categorized as idiopathic (36\%), diabetic (29\%), and postsurgical $(13 \%){ }^{1-3}$ However, the current treatment method for gastroparesis is not often very effective because it does not adequately address the underlying causes in terms of offering less invasive solutions. Instead, it involves symptom management through medications, such as prokinetics and antiemetics, dietary modifications, enteral nutrition supply, and surgical interventions such as pyloroplasty. Hence, a better alternative is necessary to overcome the limitations of such conventional methods. Gastric electrical stimulation (GES), which utilizes electrodes and a pulse generator to electrically stimulate the gastric tissues, can effectively mitigate the symptoms of gastroparesis. ${ }^{3-6}$ In clinical studies on patients with gastroparesis, GES was observed to significantly improve gastrointestinal symptoms, especially nausea and vomiting, and reduce hospitalization and use of medication, thereby enhancing the patients' quality of life. However, devices that are currently in clinical use require invasive procedures to be performed under general anesthesia. Also, this invasive procedure can induce complications such as implantation site pain, infection, dislodgement, and skin problems, and is also associated with risk of device removal. ${ }^{7}$

Therefore, a new type of device is needed for minimally invasive insertion using tools such as an endoscope. Minimally invasive endoscopic GES device implantation requires miniaturization, waterproofing, and insulation of the device, as well as external control of the pulse frequency, pulse width, and switching on/off of GES. We aim to assess the feasibility of a newly developed wireless gastric electrical stimulator and describe its minimally invasive implantation procedure via endoscopy.

\section{Materials and Methods}

\section{Gastric Electrical Stimulation Device: Prototype}

We developed a device consisting of 2 parts: a transmitter and a receiver, as shown in Figure 1A. The 2 systems were based on a microcontroller unit (8-bit MCU: CC1310F128RSM, ABOV chip; ABOV Semiconductor, Cheongju-si, Korea) with a battery-operated $(3.7 \mathrm{~V})$ transmitter. The transmitter first determined the mode and then transmitted it via radio frequency communication using a transmitting module of 868/915 MHz (ACAJ-110-T) (Fig. 1BD). The corresponding receiver $(22 \mathrm{~mm} \times 8 \mathrm{~mm}$ dimensions $)$ for
A

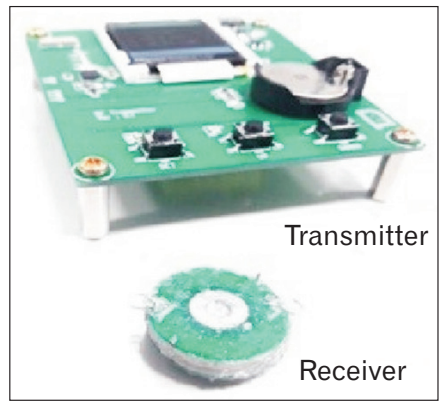

B

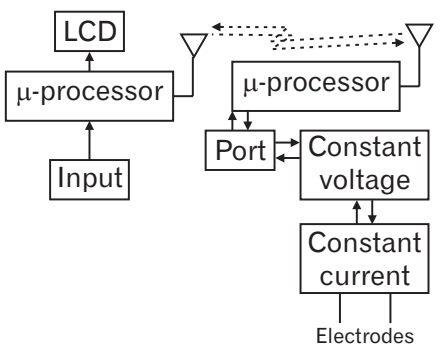

C

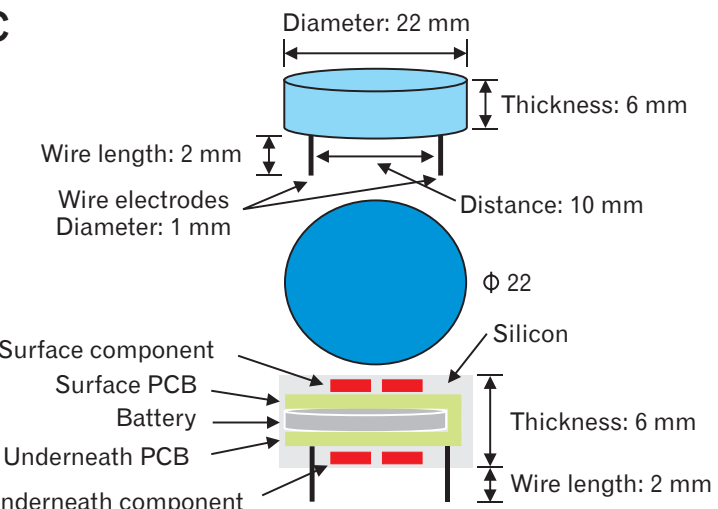

D

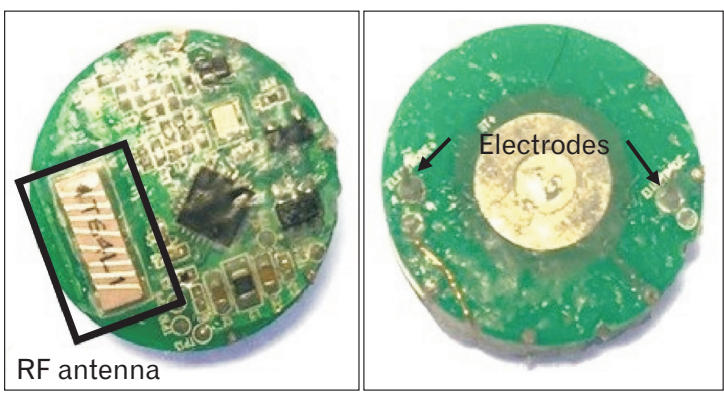

Figure 1. Gastric electrical stimulation (GES) device. (A) A transmitter and a receiver. (B) Block diagram of the system, including the signal transmitter and receiver. (C) Schematic of the system. (D) Radiofrequency (RF) antenna and electrodes of GES. PCB, printed circuit board. 
GES, which resembled a coin battery, was battery operated $(3.0 \mathrm{~V})$ and easy to transfer into the intestinal tube (Fig. 1C and 1D). The receiver consisted of the same module as the transmitter and simultaneously induced a $5 \mathrm{~mA}$ constant current stimulation via switching circuits made up of an NPN-type transistor (KTN2222AU, KEC, Seoul, Korea), a $120-\Omega$ resistor, and a timer (TPS22860DBVR, Texas Instruments, Dallas, TX, USA) that served as a clock for switching (Fig. 2A). The timer worked at frequencies of 14, 28, and $55 \mathrm{~Hz}$, with a pulse width of 1-999 microseconds, which corresponded to a duty cycle of $0.0014-5.208 \%$, and an on and off time cycle of 0.1-9.9 seconds. The system included a wire-type 2-electrode system for electrical stimulation. To verify the system (voltage and current), the outputs were checked for various loads using an oscilloscope (TDS-2002C; Tektronix, Beaverton, OR, USA) and a current probe (Tektronix p6021a, Tektronix, USA). After verification, the device was encapsulated in a protective silicone cover for waterproofing (Fig. 1D). The system consumed $20 \mathrm{~mW}$ and could operate continuously for more than 2 hours using a $3 \mathrm{~V}, 620$ milliampere hour $(\mathrm{mAh})$ coin cell battery. For electrical stimulation of the GES device, electrodes of $2 \mathrm{~mm}$ length and $1 \mathrm{~mm}$ diameter (Sus 304) made of medical-grade stainless steel were inserted into the muscle layer $10 \mathrm{~mm}$ apart. The GES device received radio frequency signals for the determined parameters (ie, frequency, duty cycle, and treatment time), and the stimulation was initiated through remote control.

\section{Slow Wave Acquisition}

A multi-channel recorder (Acknowledge 4.4, MP150; Biopac Systems, Santa Barbara, CA, USA) was used to record the gastric myoelectrical activity throughout the study. The gastric slow wave measurements were obtained through the $\mathrm{Ag} / \mathrm{AgCl}$ electrodes placed on the serosal layer of the porcine stomach. The electrodes were located at a total of 6 sites on the fundus, body, and antrum of the porcine gut. Measurements were obtained prior to electrical stimulation. During the stimulation, the recorder had to be maintained safe from electrical shock because the circuit for the measurements was repeatedly opened by disconnecting the ground line. Upon completion of the stimulation, the circuit of the recorder was closed, and the recorder commenced recording signals. The signal amplitudes were divided by the amplitude of $4 \mathrm{~Hz}$ response to attain the frequency-to-amplitude ratio (FAR) corresponding to the range of $3 \mathrm{~Hz}$ to $4 \mathrm{~Hz}$.
A

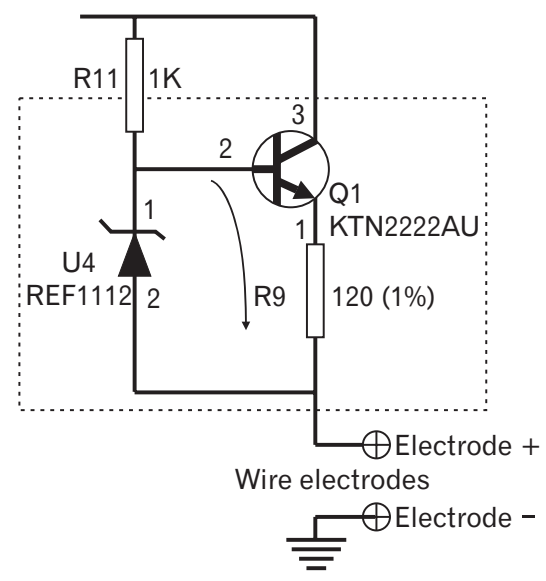

B
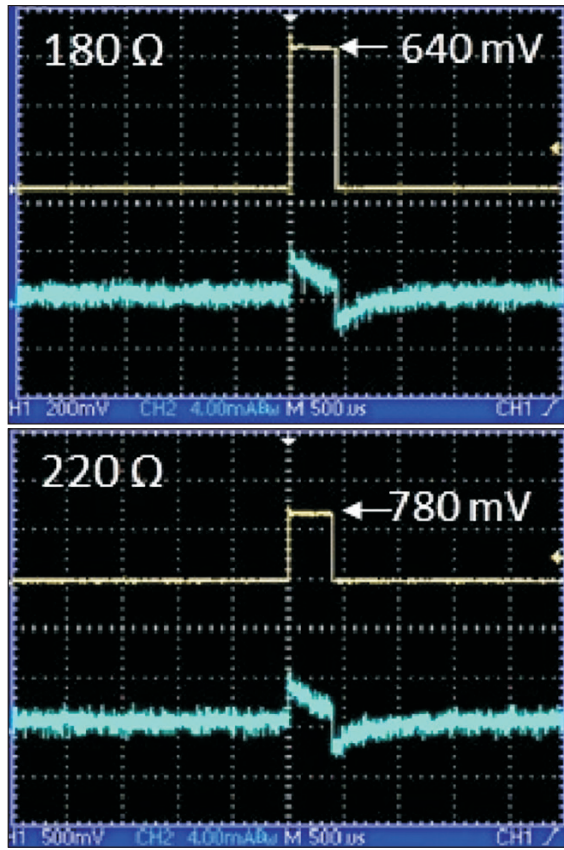
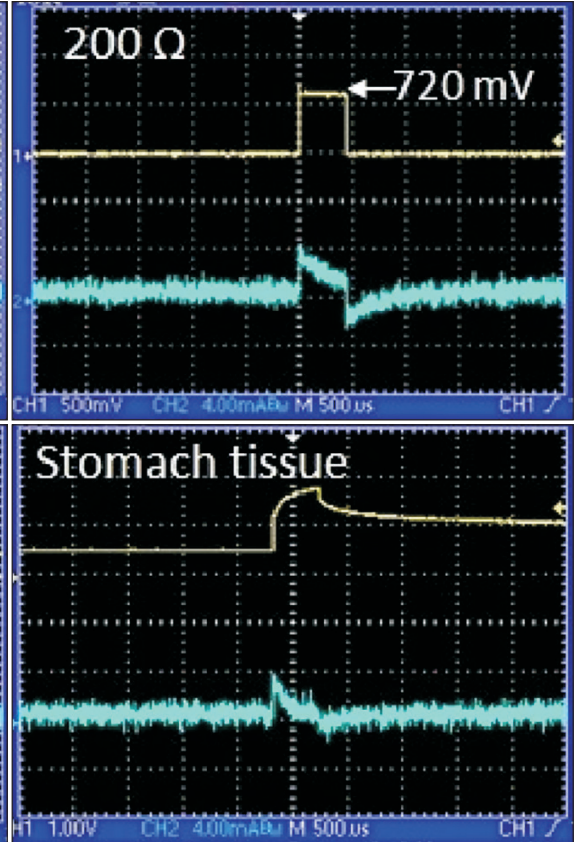

Figure 2. Circuit for constant current and performance of the constant current for various resistors. (A) The NPN (Negative-Positive-Negative)type transistor (KTN222AU) has a voltage drop of $0.7 \mathrm{~V}$ between the emitter and the base of the transistor and $5 \mathrm{~mA}$ current via the $120-\Omega$ resistor (R9). The current passes through the tissue via the electrodes. (B) The circuit showed a constant current output of approximately $5 \mathrm{~mA}$ for the 180,200 , and $220 \Omega$ resistors. The circuit showed an output of $1.6 \mathrm{~mA}$ for the stomach tissue. 


\section{Ethics Statement}

This study was performed in strict accordance with the recommendations in the Guide for the Care and Use of Laboratory Animals of the National Institutes of Health. The study protocol was approved by the Committee on the Ethics of Animal Experiments of the Korea University Anam Hospital (Permit No. KOREA-2018-0023). All efforts were made to minimize animal suffering.

\section{Anesthesia and Endoscopic Placement of Device}

The animal study was performed under general anesthesia that was induced with azaperone (2-8 $\mathrm{mg} / \mathrm{kg})$ and xylazine $(1-3 \mathrm{mg} /$ $\mathrm{kg})$ and maintained with isoflurane (1.5-2\%) on pigs weighing 40 $50 \mathrm{~kg}$, followed by a laparotomy. Endoscopy was then performed to install the GES device.

To transport the GES device to the stomach, a guidewire was directed along the porcine upper gastrointestinal tract, and the GES device was delivered wrapped in a small rubber pocket using rat tooth forceps. After the GES device delivery, the submucosal layer was removed as deeply as possible. A pocket was then made for embedding the GES. Thereafter, the GES electrode was placed in

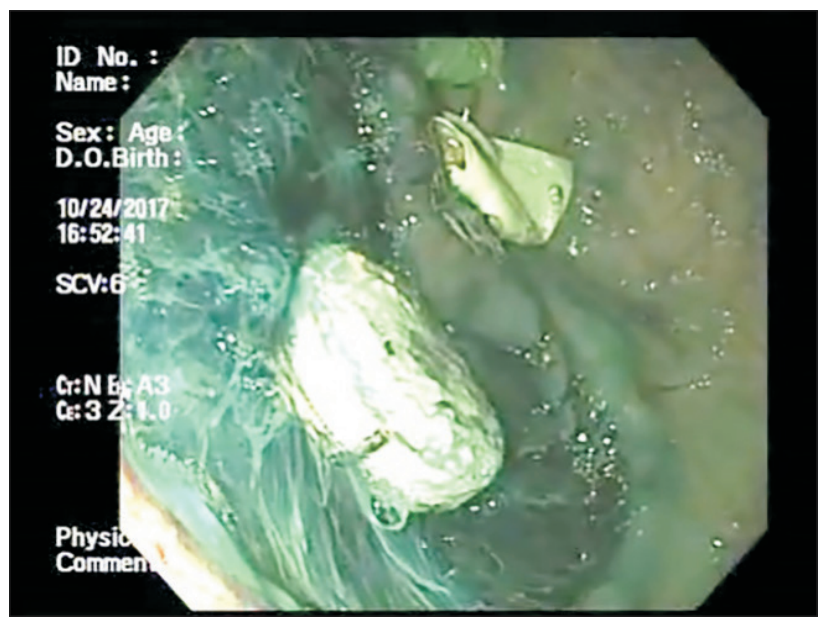

Figure 3. Endoscopic implantation of the gastric electrical stimulation device. contact with the muscularis propria for electrical stimulation and was fixed with an endoscopic clip (Fig. 3).

\section{Electrical Stimulation Protocol}

After an hour of resting time following the endoscopic submucosal dissection procedure, electrical stimulation was conducted for 10 minutes at a frequency of $14 \mathrm{~Hz}$ with a pulse width of 330 microseconds, which corresponded to a duty cycle of $0.459 \%$. The pulse had cycle on and off times of 0.1 seconds and 5.0 seconds, respectively. The gastric slow wave signals were then obtained in 3 steps: baseline, stimulation, and recovery period for 10 minutes, in each stage.

\section{Results}

The GES device was configured to receive signals through a $200-\Omega$ resistor through a phantom serosa-like tissue, including 180 and $200-\Omega$ resistors to confirm signals as commands. ${ }^{8}$ The output voltage and current waveforms for a pulse width of 330 microseconds at a frequency of $14 \mathrm{~Hz}$ are shown in Figure 2B. Varying the load value via a resistor, the current outputs at both electrodes were constant at approximately $5 \mathrm{~mA}$ and $1.6 \mathrm{~mA}$ for the stomach tissue

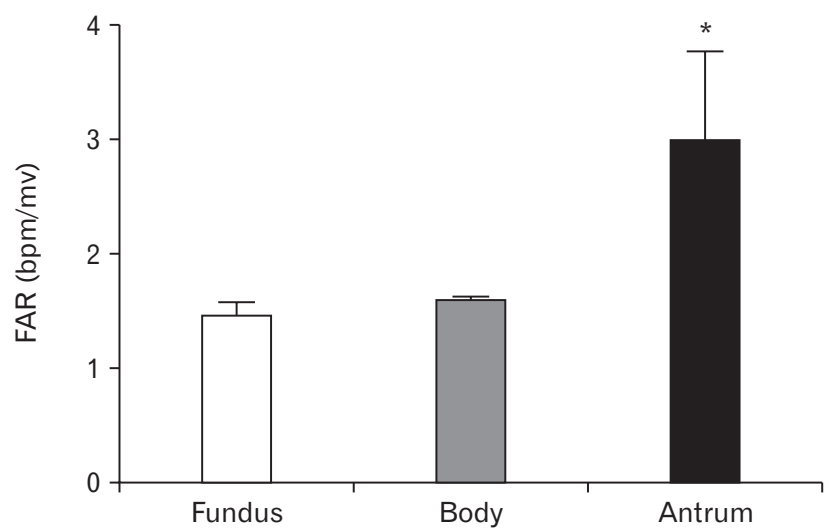

Figure 4. Frequency-to-amplitude ratio (FAR) after electrical stimulation, according to the different lesions. ${ }^{*} P<0.05$ compared to the fundus or body lesion. bpm, beats per minute.

Table. Summary of the Measured Electrogastrogram

\begin{tabular}{lccccc}
\hline \multicolumn{1}{c}{ Item } & $\begin{array}{c}\text { Frequency range } \\
(\mathrm{bpm})\end{array}$ & $\begin{array}{c}\text { Mean frequency } \\
(\mathrm{bpm})\end{array}$ & $\begin{array}{c}\text { Amplitude } \\
\text { type }\end{array}$ & $\begin{array}{c}\text { Amplitude range } \\
(\mathrm{mV})\end{array}$ & $\begin{array}{c}\text { FAR } \\
(\mathrm{bpm} / \mathrm{mV})\end{array}$ \\
\hline Baseline & $2.00-4.00$ & 2.54 & $\mathrm{UA}$ & $0.052-0.420$ & 1.5 \\
Stimulation by a wireless GES device & $3.00-4.00$ & 3.26 & EA & $0.103-0.230$ & 5.9 \\
After stimulation & $3.00-5.00$ & 3.00 & $\mathrm{UA}$ & $0.042-0.152$ & 3.0 \\
\hline
\end{tabular}

bpm, beats per minute; FAR, frequency-to-amplitude ratio; GES, gastric electrical stimulation; UA, unequal amplitude; EA, equal amplitude. 


\section{A Before stimulation}
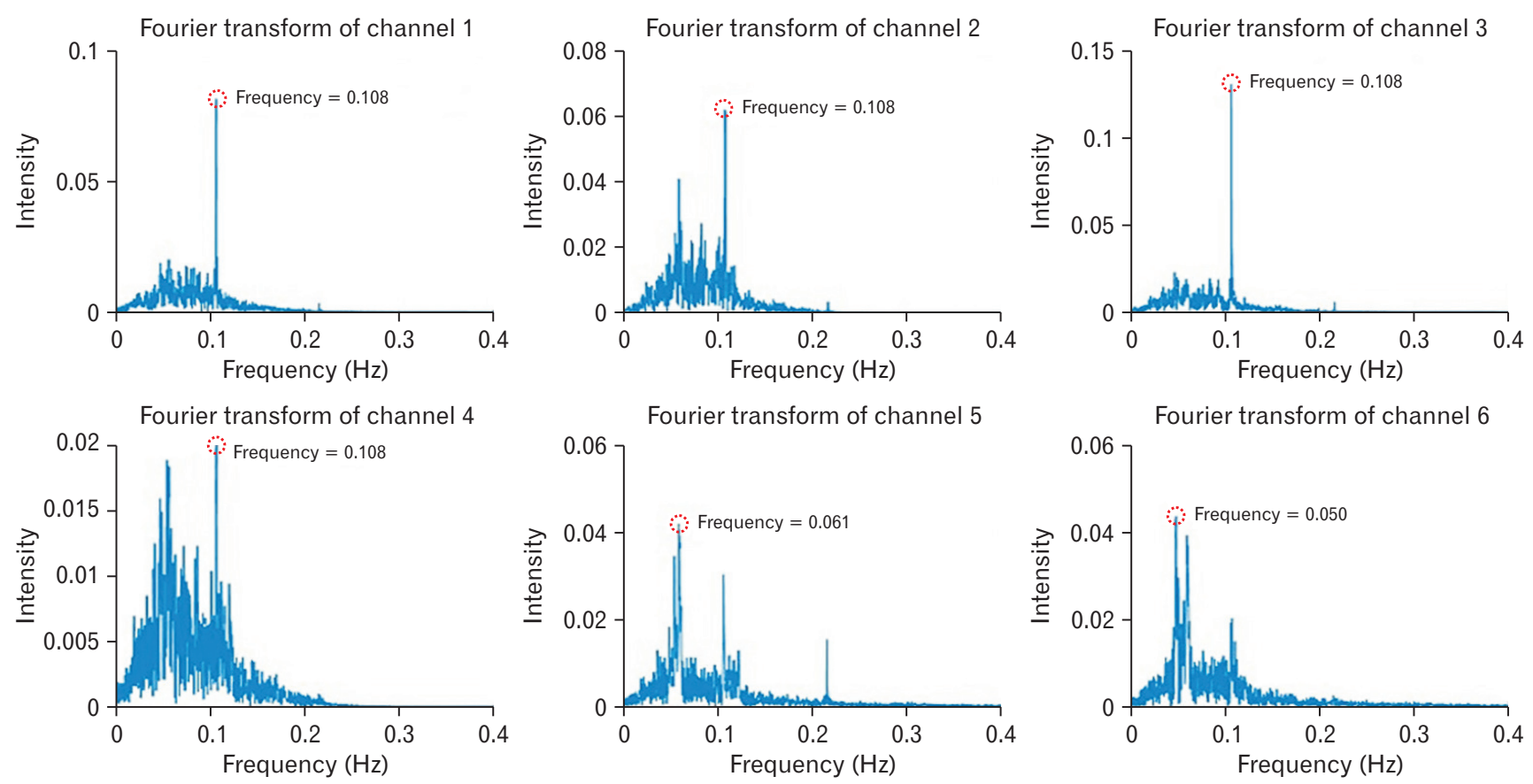

\section{B After stimulation}
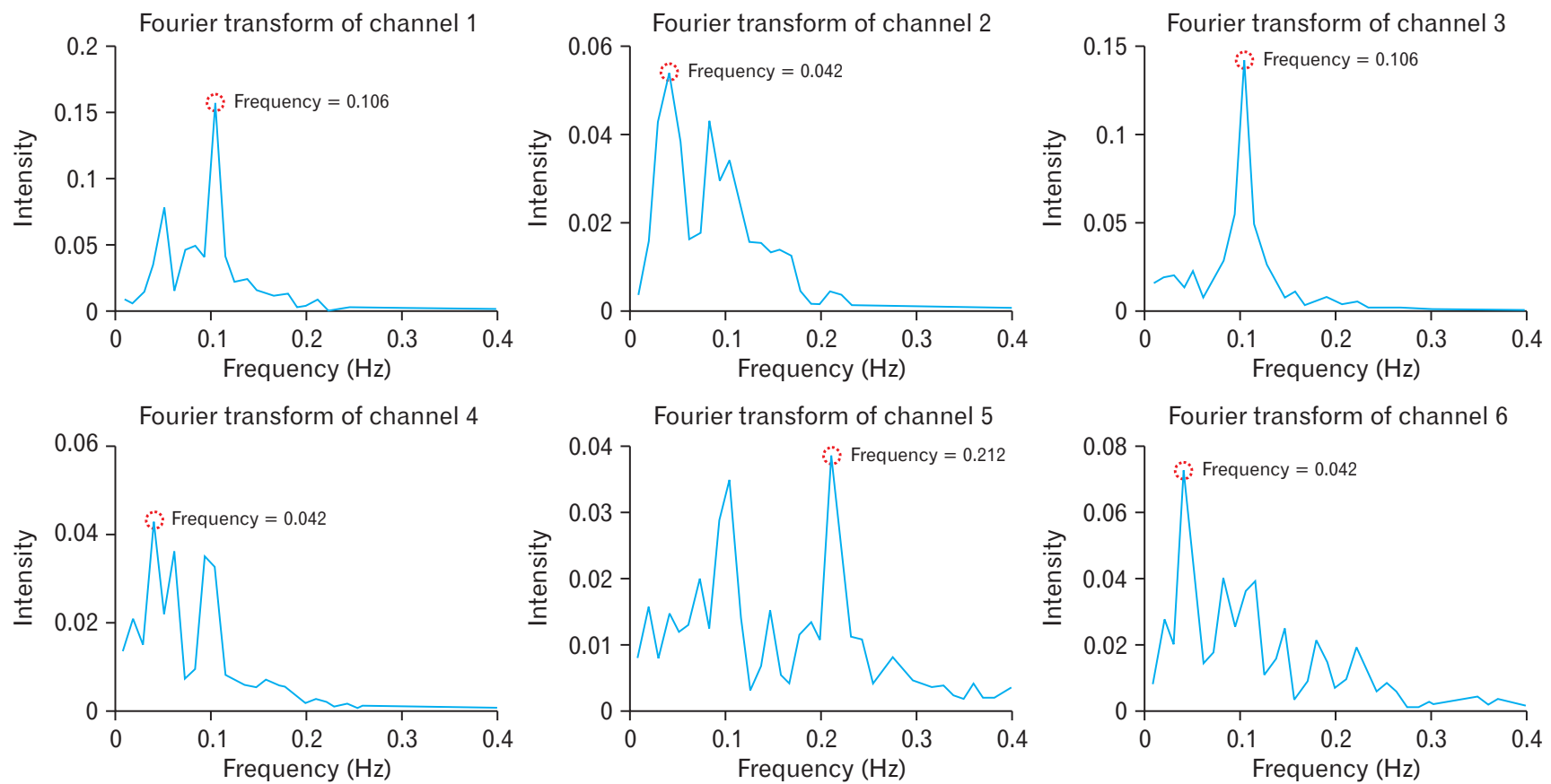

Figure 5. Fourier analysis of the gastric slow wave (fundus: channels 1 and 2; body: channels 3 and 4; and antrum: channels 5 and 6). (A) Fourier transform wave pattern before electrical stimulation. (B) Fourier transform wave pattern after electrical stimulation.

(Fig. 2B).

The FAR was calculated based on the data collected (Table). The FAR varied depending on the site (Fig. 4). The antrum showed a significant increase in FAR compared to the fundus or body lesion $(P<0.05)$ (Fig. 4), despite all sites receiving the same amount of electrical stimulation. Fourier transforms (FT) were per- 
formed on the acquired signals to analyze the frequency dependence of myoelectrical activity. The Games-Howell variance analysis of FT showed that the slow wave frequency tended to increase in the frequency domain after electrical stimulation at the antrum compared to the fundus and the body, shifting from $0.061 \mathrm{~Hz}$ to 0.212 $\mathrm{Hz}$. The slow wave intensity tended to increase in the body and the fundus compared to the antrum (Fig. 5).

\section{Discussion}

In this study, we developed a wireless GES device and inserted it into the gastrointestinal tracts of weaner pigs via an endoscope, presenting a possible new method for insertion of a GES device without invasive surgery. Stimulation was applied to the gastric muscles through a pair of electrodes, with either constant-voltage or constant-current control. This electrical stimulation was applied as electric impulses, which mimicked the action potentials from the central nervous system, causing the muscles to contract. An action potential is made by muscle cell depolarization. Depolarization occurs when existing ionic charges change to opposite charges. The ions respond to the electrons emitted by either the positive or negative electrode. With this action, the constant-current stimulation allows the power developed by the muscle to be better controlled, while the constant-voltage is not sufficient to exert around the entire tissue between the electrodes due to the heterogeneous impedance of the tissue. We applied the constant-current control herein. In the laboratory test, the GES outcome was a constant current of $5 \mathrm{~mA}$ for loads of 180,200 , and $220 \Omega$. In the stomach tissue test, the output was $1.6 \mathrm{~mA}$ and did not approach the $5 \mathrm{~mA}$ value of a fixed resistor. The embedded GES device was able to communicate bidirectionally with the transmitter outside the body, although the device was embedded in the pig model. This outcome was similar to that of a previous study, ${ }^{9}$ perhaps due to the passive film that formed on the electrode surface when inserted into the tissue. ${ }^{10}$

GES applied to the mucosal tissue showed a significant increase in the FAR at the antrum compared to that at the fundus or body, when GES was applied on the body. The FAR generally reflected the tendency of depending on frequency for objects. ${ }^{11}$ In other words, the antrum responded more sensitively to the electrical stimulation than the others. Frequency is defined as the number of vibrations undergone during one unit of time by a body in periodic motion. The vibration is related with the elastic properties of the stomach tissue. Among the structures of the stomach, the antrum showed strong elasticity assuming each part of the stomach has all mass and amplitude.
The FAR results reflected the FT analysis of the frequency of the gastric myoelectrical activity post-stimulation, which refers to the frequency domain representation. FT of the antrum showed a significant increase in frequency of 0.151 compared to that before stimulation, while the others exhibited no changes in frequency between before and after electrical stimulation in channel 5 . However, in channels 2 and 4, FT of the fundus and the body showed a decrease of 0.066 , but was absent in the antrum. In channels 2 and 4, the frequent frequency of FT was within the domain of $0.1 \mathrm{~Hz}$, despite the frequency shift. However, in the case of the antrum, the shift of the FT frequency was significant, reflecting the propagation of the electrical slow wave from the proximal stomach toward the antrum and the pylorus. ${ }^{12}$ In addition, this may be attributed to the interstitial cells of Cajal (ICC) in the antrum tissue. According to previous studies, ${ }^{13,14}$ the ICC are more densely located in the corpus and antrum than in the fundus. The antrum contains both ICC of the myenteric plexus and intramuscular ICC networks, whereas the fundus only contains intramuscular ICC. The ICC of the myenteric plexus serve as a pacemaker, which creates the bioelectrical slow wave potential that leads to the smooth muscle contraction. It is likely to correlate between frequent frequency increases in 0.151 $\mathrm{Hz}$ and ICC in the antrum tissue.

The limitation of this study is that no result was obtained regarding the use of constant-voltage in a pig model. We could not compare the results from constant-voltage and constant-current conditions. Also, in the pig model used, we did not conduct a valuation of gastric function, assessment of the device in an animal model with functional dyspepsia, or a histological analysis of the ICC. Nevertheless, the present study has strength in terms of providing insights into applying a pacemaker to the stomach via an endoscope. We tried to evaluate the effect of electrical stimulation by presenting a change in the slow wave rhythmic activity.

In conclusion, GES is feasible with our minimally invasive wireless device. This technique could overcome the limitations of invasive conventional procedures and has the potential to increase utilization of GES as a treatment alternative.

Financial support: This work was supported by the National Research Foundation of Korea (NRF) grant funded by the Korean Government (Grant No. NRF-2019R1C1C1009819) and by the Ministry of Trade, Industry and Energy (MOTIE, Korea) under the Industrial Technology Innovation (Program No. 10060251, "Development of diagnostic device for functional dyspepsia based on Korean-Western medicine fusion abdominal diagnosis").

\section{Conflicts of interest: None.}


Author contributions: Seung Han Kim and Hoon Jai Chun conceived and designed the experiments; Hyuk Soon Choi and Hong Bae Kim designed the experimental methodologies; Seung Han Kim, Hong Bae Kim, and Hyuk Soon Choi ran experiments and collected data; Eun Sun Kim, Bora Keum, Yeon Seok Seo, Yoon Tae Jeen, and Soon Ho Um analyzed the results; Hoon Jai Chun, Hong Sik Lee, and Chang Duck Kim supervised the study; Seung Han Kim and Hong Bae Kim wrote the draft; and Hyuk Soon Choi revised the manuscript. All authors contributed to writing, editing, and review of the manuscript.

\section{References}

1. Hyett B, Martinez FJ, Gill BM, et al. Delayed radionucleotide gastric emptying studies predict morbidity in diabetics with symptoms of gastroparesis. Gastroenterology 2009;137:445-452.

2. Camilleri M, Parkman HP, Shafi MA, Abell TL, Gerson L; American College of Gastroenterology. Clinical guideline: management of gastroparesis. Am J Gastroenterol 2013;108:18-37.

3. Liu N, Abell T. Gastroparesis updates on pathogenesis and management. Gut Liver 2017;11:579-589.

4. Navas CM, Patel NK, Lacy BE. Gastroparesis: medical and therapeutic advances. Dig Dis Sci 2017;62:2231-2240.

5. Singh S, McCrary J, Kedar A, et al. Temporary endoscopic stimulation in gastroparesis-like syndrome. J Neurogastroenterol Motil 2015;21:520527.
6. Klinge MW, Rask P, Mortensen LS, et al. Early assessment of costeffectiveness of gastric electrical stimulation for diabetic nausea and vomiting. J Neurogastroenterol Motil 2017;23:541-549.

7. Shen S, Luo H, Vachaparambil C, et al. Gastric peroral endoscopic pyloromyotomy versus gastric electrical stimulation in the treatment of refractory gastroparesis: a propensity score-matched analysis of long term outcomes. Endoscopy 2020;52:349-358.

8. Abell T, McCallum R, Hocking M, et al. Gastric electrical stimulation for medically refractory gastroparesis. Gastroenterology 2003;125:421428.

9. Deb S, Tang SJ, Abell TL, et al. An endoscopic wireless gastrostimulator (with video). Gastrointest Endosc 2012;75:411-415, e1.

10. Zhang L, Duan Y, Gao R, et al. The effect of potential on surface characteristic and corrosion resistance of anodic oxide film formed on commercial pure titanium at the potentiodynamic-aging mode. Materials (Basel) 2019;12:370.

11. Singh NK, Barman A. Frequency-amplitude ratio of ocular vestibularevoked myogenic potentials for detecting Meniere's disease: a preliminary investigation. Ear Hear 2016;37:365-373.

12. Lammers WJ. Inhomogeneities in the propagation of the slow wave in the stomach. Neurogastroenterol Motil 2015;27:1349-1353.

13. Mazet B, Raynier C. Interstitial cells of cajal in the guinea pig gastric antrum: distribution and regional density. Cell Tissue Res 2004;316:23-34.

14. Hirst GD, Beckett EA, Sanders KM, Ward SM. Regional variation in contribution of myenteric and intramuscular interstitial cells of cajal to generation of slow waves in mouse gastric antrum. J Physiol 2002;540(Pt 3):1003-1012. 